Campelo, FSAA, BritoJúnior, WV \& Veloso, LC (2020). Epidemiological profile of syphilis cases in pregnant women from 2014 to 2018 in the state of Piauí. Research, Society and Development, 9(7): 1-11, e488974382.

\title{
Perfil epidemiológico dos casos de sífilis em gestantes de 2014 a 2018 no estado do Piauí Epidemiological profile of syphilis cases in pregnant women from 2014 to 2018 in the state of Piauí
}

Perfil epidemiológico de casos de sífilis en mujeres embarazadas de 2014 a 2018 en el estado de Piauí

Recebido: 10/05/2020 | Revisado: 11/05/2020 | Aceito: 13/05/2020 | Publicado: 23/05/2020

\author{
Francisco Samuel Andrade de Alencar Campelo \\ ORCID: https://orcid.org/0000-0001-8182-5044 \\ Centro Universitário Santo Agostinho, Brasil \\ E-mail: samuel.alencarr@gmail.com
}

Walber de Vasconcelos Brito Júnior

ORCID: https://orcid.org/0000-0002-3439-4592

Centro Universitário Santo Agostinho, Brasil

E-mail: walber-vasconcelos@hotmail.com

Laurimary Caminha Veloso

ORCID: https://orcid.org/0000-0002-3245-6307

Centro Universitário Santo Agostinho, Brasil

E-mail: laurimary.caminha@gmail.com

\section{Resumo}

No Brasil, a sífilis tem se apresentado como uma epidemia pela sua grande incidência, e tratase de uma patologia infectocontagiosa que vem sendo negligenciada na atenção à saúde. Quando diagnosticada na gestação denomina-se sífilis gestacional, e pode evoluir para parto prematuro, aborto espontâneo, óbito perinatal e sífilis congênita. O objetivo do artigo é descrever o perfil epidemiológico dos casos notificados de sífilis em gestantes, entre 2014 e 2018 no Estado do Piauí a partir de dados do Sistema Nacional de Agravos de Notificação (SINAN). Trata-se de uma pesquisa epidemiológica, documental, retrospectiva, de caráter descritivo, com abordagem quantitativa. A coleta dos dados equivale aos casos de sífilis em gestantes notificados no SINAN, no período de 2014 a 2018 no Estado do Piauí, sendo estes disponibilizados pelo Departamento de Informática do SUS (DATASUS), tabulados pelo 
TABNET e processados no Microsoft Excel. Os dados foram analisados segundo as variáveis socioculturais e clínicas das gestantes: faixa etária, nível de escolaridade, idade gestacional, esquema de tratamento e classificação clínica. Nos resultados obtidos foram notificados 1.954 casos de sífilis em gestantes. Prevaleceu a faixa etária entre os 20 e 29 anos (49,3\%). O $3^{\circ}$ trimestre gestacional apresentou o maior número de notificações 808 (41,3\%). Quanto à classificação clínica, prevaleceu a forma latente com 646 casos (33\%). Conclui-se que nos últimos anos vem ocorrendo um aumento das notificações de sífilis em gestantes no Piauí, e que o estado necessita de apoio e planejamento para a adesão de estratégias de controle e erradicação dessa condição evitável e tratável.

Palavras-chave: Sífilis; Infecções sexualmente transmissíveis; Gestação.

\section{Abstract}

In Brazil, the syphilis has presented itself as an epidemic due to its high incidence, and it is an infectious disease but that has been neglected in health care. When diagnosed in pregnancy it is called gestational syphilis, and it can progress to premature birth, spontaneous abortion, perinatal death and congenital syphilis. The objective of the article is to describe the epidemiological profile of notified cases of syphilis in pregnant women between 2014 and 2018 in the State of Piauí using data from the National Notifiable Diseases Surveillance System (NNDSS). This is an epidemiological, documentary, retrospective, descriptive study with a quantitative approach. The data collection is equivalent to the cases of syphilis in pregnant women notified in the NNDSS, from 2014 to 2018 in the State of Piauí, these being made available by the SUS Computer Department (DATASUS), tabulated by TABNET and processed in Microsoft Excel. The data was analyzed according to the socio-cultural and clinical variables of the pregnant women: age group, educational level, gestational age, treatment scheme and clinical classification. In the results obtained, 1.954 cases of syphilis were reported in pregnant women. The age group between 20 and 29 years old prevailed $(49.3 \%)$. The $3 \mathrm{rd}$ gestational trimester presented the highest number of notifications $808(41.3 \%)$. As for the clinical classification, the latent form prevailed with 646 cases (33\%). Concludes that in recent years there has been an increase in notifications of syphilis in pregnant women in Piauí, and that the state needs support and planning for the adhesion of strategies to control and eradicate this preventable and treatable condition.

Keywords: Syphilis; Sexually transmitted infections; Pregnancy. 


\section{Resumen}

En Brasil, la sífilis se ha presentado como una epidemia debido a su alta incidencia, y es una enfermedad infecciosa pero que se ha descuidado en la atención médica. Cuando se diagnostica en el embarazo, se llama sífilis gestacional, y puede progresar a un parto prematuro, aborto espontáneo, muerte perinatal y sífilis congénita. El propósito del artículo es describir el perfil epidemiológico de los casos notificados de sífilis en mujeres embarazadas entre 2014 y 2018 en el estado de Piauí utilizando datos del Sistema Nacional de Vigilancia de Enfermedades de Notificación (SNVEN). Este es um estudio epidemiológico, documental, retrospectivo, descriptivo con enfoque cuantitativo. La recopilación de datos es equivalente a los casos de sífilis en mujeres embarazadas notificadas en el SNVEN, de 2014 a 2018 en el Estado de Piauí, que están disponibles por el Departamento de Computación del SUS (DATASUS), tabulado por TABNET y procesado en Microsoft Excel. Los datos se analizaron según las variables socioculturales y clínicas de las mujeres embarazadas: grupo de edad, nivel educativo, edad gestacional, esquema de tratamiento y clasificación clínica. En los resultados obtenidos, se reportaron 1.954 casos de sífilis en mujeres embarazadas. 1.954 casos de sífilis en mujeres embarazadas. Prevaleció el grupo de edad entre 20 y 29 años (49,3\%). El tercer trimestre de gestación presentó el mayor número de notificaciones 808 (41,3\%). En cuanto a la clasificación clínica, la forma latente prevaleció con 646 casos (33\%). Concluye que en los últimos años ha habido un aumento en las notificaciones de sífilis en mujeres embarazadas en Piauí, y que el estado necesita apoyo y planificación para la adhesión de estrategias para controlar y erradicar esta condición prevenible y tratable.

Palabras clave: Sífilis; Infecciones de transmisión sexual; Embarazo.

\section{Introdução}

Atualmente, as Infecções Sexualmente Transmissíveis (IST) são consideradas no mundo, um dos grandes problemas de saúde pública. No Brasil, a sífilis tem se apresentado como uma epidemia pela grande incidência, e que vem sendo negligenciado na atenção à saúde.

A sífilis é uma patologia infectocontagiosa, de fácil diagnóstico e tratamento de baixo custo. Seu agente etiológico causador é o Treponema Pallidum, uma bactéria transmitida pelo contato sexual, com sangue infectado ou por transmissão vertical. É uma doença que apresenta fases de agudização podendo tornar-se crônica acometendo diversos órgãos e 
sistemas do corpo, e levar à morte se não tratada a tempo. Quando diagnosticada na gestação denomina-se sífilis gestacional (Brasil, 2019).

Segundo o Ministério da Saúde, a sífilis apresenta cerca de 6 milhões de novos casos a cada ano em todo o mundo, e no Brasil a população mais afetada são as mulheres, principalmente as negras e jovens, entre faixa etária de 20 a 29 anos, e é evidente que as mulheres mais acometidas pela sífilis encontram-se em idade fértil, o que eleva os riscos para o desenvolvimento da doença na gestação, resultando no aumento dos índices de infecção em mulheres grávidas (Brasil, 2018).

O tratamento da sífilis não difere do período gestacional para os casos de sífilis adquirida, a medicação de primeira escolha é a Penicilina $\mathrm{G}$ benzatina. O diagnóstico e adesão ao tratamento da mulher com sífilis no período gravídico ocorrem por meio da atenção prénatal, que além de oferecer uma atenção integral no intuito de curá-la da infecção, também visa prevenir a sífilis congênita do seu concepto (Vasconcelos, 2017).

Diante dessa problemática, percebe-se que a sífilis na gestação trata-se de um desafio para saúde pública a ser enfrentado, no qual se consiste em detectar precocemente e tratar a doença, contribuir na diminuição da morbimortalidade materno-fetal, e adotar estratégias e medidas para que ocorra a melhoria dos indicadores de saúde. Com isso, o presente trabalho tem o objetivo de descrever o perfil epidemiológico dos casos notificados de sífilis em gestantes, entre 2014 e 2018 no Estado do Piauí a partir de dados do Sistema Nacional de Agravos de Notificação (SINAN).

\section{Metodologia}

Trata-se de uma pesquisa epidemiológica, documental, retrospectiva, de caráter descritivo, com abordagem quantitativa sobre taxas de sífilis em gestantes encontradas no estado do Piauí no período de 2014 e 2018. Os dados foram obtidos pelo Sistema de Informações no banco de dados oficial do SINAN, do Departamento de Informática do Sistema Único de Saúde (DATASUS).

A coleta dos dados equivale aos casos de sífilis em gestantes notificados no Sistema de Informação de Agravos de Notificações (SINAN), no período de 2014 a 2018 no Estado do Piauí, sendo estes disponibilizados pelo Departamento de Informática do SUS (DATASUS), tabulados pelo TABNET e processados no Microsoft Excel. O programa TabWin do DATASUS/Ministério da Saúde foi utilizado para analisar os dados segundo as variáveis 
socioculturais e clínicas das gestantes: faixa etária, nível de escolaridade, idade gestacional, esquema de tratamento e classificação clínica.

Foram incluídos neste estudo todos os dados de casos que foram notificados nos anos de 2014 a 2018. Como critérios de exclusão do estudo os dados registrados fora do contexto do estudo.

Considerando as características da pesquisa, assegura-se que os riscos são mínimos visto que os dados foram coletados no banco de dados oficial do SINAN. Os pesquisadores garantiram que foi mantida a confidencialidade das informações contidas no SINAN, a privacidade, o anonimato dos participantes para que as informações fossem utilizadas sem causar nenhum prejuízo. Os benefícios serão indiretos, e associados ao levantamento de informações que possam contribuir para o redirecionamento de ações melhorando a questão de políticas públicas voltadas para o enfrentamento da sífilis.

\section{Resultados}

No Piauí, foram notificados 1.954 casos entre os anos de 2014 e 2018 de Sífilis em Gestantes no Sistema de Informação de Agravos e Notificação (SINAN). Observa-se que durante o período estudado houve um comportamento crescente nos números de casos por ano cuja taxa de incidência foi numa média de 8,08/000 hab. Em 2014 ocorreram 206 casos (10,5\%), em 2015 elevou-se para 256 (13,1\%), em 2016 foram 315 (16,1\%), em 2017447 $(22,8 \%)$ e, por fim, em 2018 foram 730 notificações (37,3\%). No Gráfico 1, pode-se observar os casos notificados de sífilis em gestantes no Piauí no período de 2014 a 2018.

Gráfico 1 - Número de casos notificados de sífilis em gestantes entre 2014 e 2018. Piauí, Brasil, 2020.

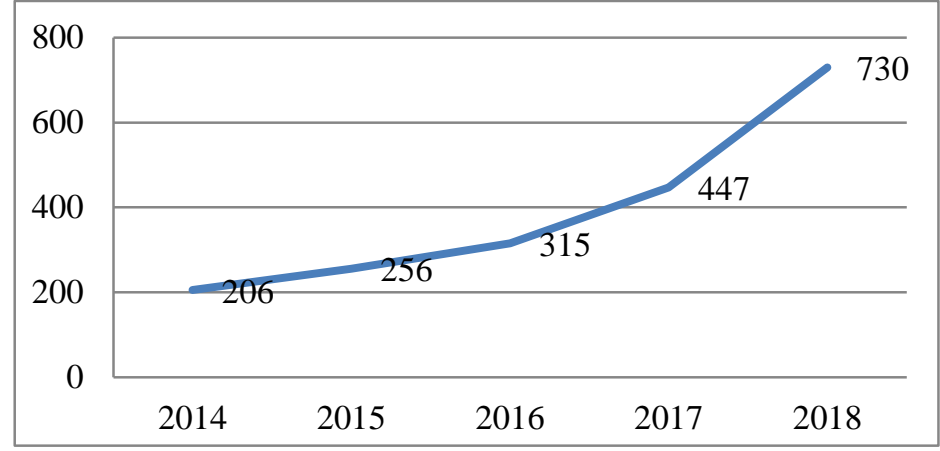

Fonte: Datasus/SINAN, 2020. 
Research, Society and Development, v. 9, n. 7, e488974382, 2020

(CC BY 4.0) | ISSN 2525-3409 | DOI: http://dx.doi.org/10.33448/rsd-v9i7.4382

Examinando o Gráfico 2 observa-se que em relação à idade das gestantes desta pesquisa, prevaleceu a faixa etária dos 20 aos 29 anos (49,3\%), logo depois 15 a 19 anos com 548 casos $(28 \%)$ possivelmente justificada pelo fato dessas mulheres encontrarem-se em idade fértil e sexualmente ativa elevando assim o risco de infecção por Sífilis na gestação.

Gráfico 2 - Total de casos notificados de gestantes com sífilis segundo faixa etária entre 2014 e 2018. Piauí, Brasil, 2020.

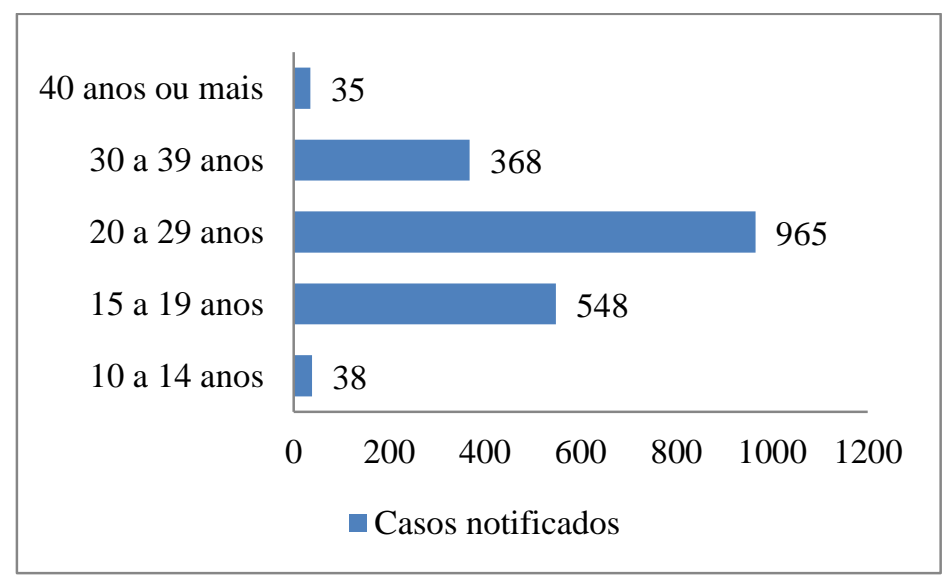

Fonte: Datasus/SINAN, 2020.

No item escolaridade, predominaram 490 gestantes (25\%) com grau de instrução de $5^{\mathrm{a}}$ a $8^{\text {a }}$ série incompleta, como mostra o Gráfico 3.

Gráfico 3 - Total de casos notificados de gestantes com sífilis segundo a escolaridade entre 2014 e 2018. Piauí, Brasil, 2020.

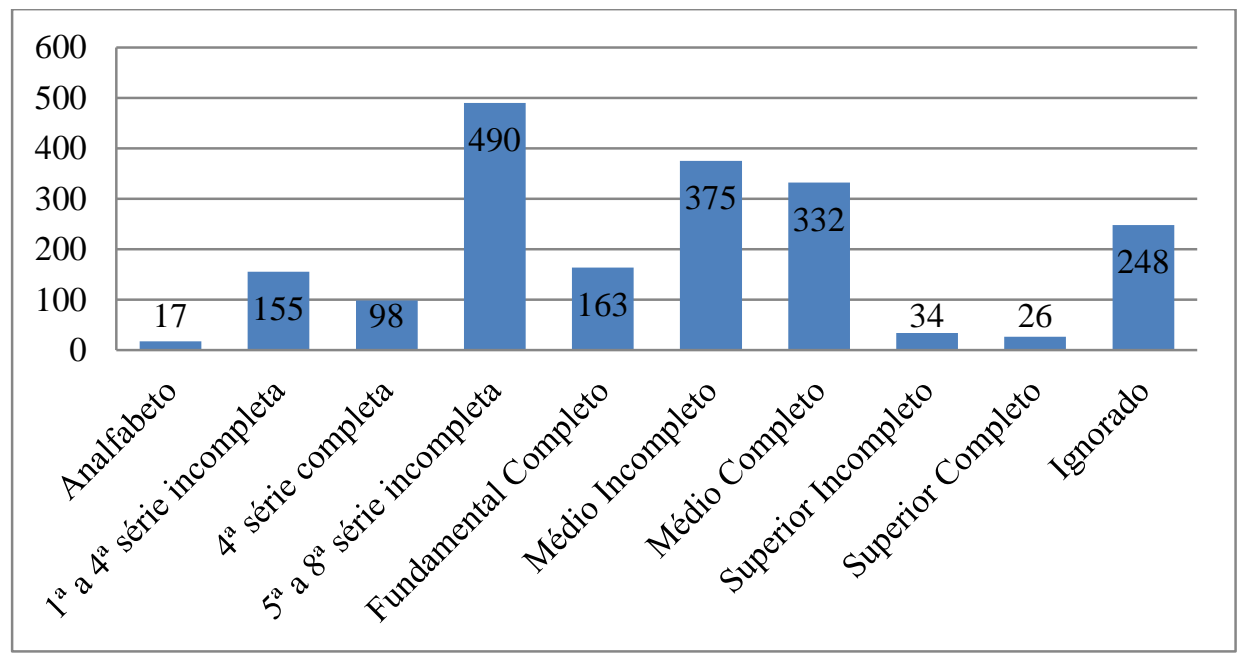

Fonte: Datasus/SINAN, 2020. 
Dentre os 1.954 casos de Sífilis em Gestantes notificados no período de 2014 a 2018, o maior grupo de gestantes, $808(41,3 \%)$ estavam no $3^{\circ}$ trimestre. No que se refere à classificação clínica, prevaleceu a forma latente, com 646 casos (33\%). O esquema de tratamento mais utilizado foi Penicilina 1603 (91,7\%), e a pesquisa também mostra que 100 gestantes não tiveram tratamento realizado $(5,7 \%)$. Na Tabela 1, é possível notar os resultados segundo a Idade Gestacional, classificação clínica e esquema de tratamento prescrito dos casos notificados de Sífilis em Gestantes.

Tabela 1 - Distribuição das características gestacional e assistencial dos casos notificados de sífilis em gestantes entre 2014 e 2018, conforme idade gestacional, classificação clínica e esquema de tratamento adotado. Piauí, Brasil, 2020.

\begin{tabular}{ccc}
\hline Características & $\mathbf{N}$ & $\mathbf{( \% )}$ \\
\hline Idade Gestacional & 425 & \\
$1^{\circ}$ Trimestre & 662 & 21,7 \\
$2^{\circ}$ Trimestre & 808 & 33,8 \\
$3^{\circ}$ Trimestre & 59 & 41,3 \\
Idade gestacional ignorada & & 3 \\
\hline Classificação Clínica & 533 & \\
Sífilis Primária & 105 & 27,2 \\
Sífilis Secundária & 322 & 5,3 \\
Sífilis Terciária & 646 & 16,4 \\
Sífilis Latente & 348 & 33 \\
Ignorado & & 17,8 \\
\hline Esquema de Tratamento & 1.603 & \\
Penicilina & 19 & 91,7 \\
Outro Esquema & 100 & 1 \\
Não realizado & 26 & 5,7 \\
Ignorado & & 1,4 \\
\hline
\end{tabular}

Fonte: Datasus/SINAN, 2020.

\section{Discussão}

De acordo com os dados descritos neste estudo é possível observar que as taxas de detecção de sífilis em gestantes no Piauí apresentaram uma tendência de aumento de 10,5\% para 37,3\% entre 2014 e 2018. Nota-se que essa tendência se dá de modo semelhante em outros estados do país, como no Amazonas e Rio de Janeiro, em pesquisa similar (Saraceni \& Miranda, 2012). Dados alarmantes que, segundo Saraceni, Pereira, da Silveira, Araújo, e Miranda (2017), mesmo com toda a facilidade e os avanços tecnológicos para o diagnóstico e o tratamento da gestante, o controle da sífilis na gestação ainda se caracteriza como um 
grande desafio para a atenção pré-natal, fato notável pelo grande número de casos da doença que aumenta cotidianamente.

A sífilis em gestantes é considerada uma doença de notificação compulsória, sendo esta notificação obrigatória em todo território nacional que foi instituída mediante a Portaria $n^{\circ}$ 33, de 14 de julho de 2005 (Brasil, 2005). Dado a magnitude desse problema, é importante a adequada notificação para o controle da sífilis, de modo que possibilite a adoção e avaliação de estratégias e medidas para que ocorra a melhoria dos indicadores de saúde.

$\mathrm{Na}$ presente pesquisa, foi observada a prevalência de mulheres com idade entre 20 e 29 anos, totalizando assim 49,3\%, em segundo, com 28\%, as mulheres com idade de 15 a 19 anos, estes achados corroboram com os achados observados em outro estudo realizado no estado de São Paulo entre 2015 a 2017, que mostra o maior número de casos de sífilis em gestante em mulheres com idade inferior a 30 anos (Jesus, 2019). Número esses, ocasionados devido se encontrarem em idade fértil e com vida sexual ativa, tornando-se assim mais suscetíveis as IST's. Observa-se a importância da consulta ativa e medidas voltadas para esse grupo, como a testagem rápida em qualquer oportunidade de disposição dessas mulheres a quaisquer serviços de saúde, que se pontua como uma ferramenta fundamental no controle da sífilis em gestante.

Outro ponto importante pode ser destacado pelo início precoce das atividades sexuais, ressaltado pelos dados de $28 \%$, onde se apresentam mulheres dos 15 a 19 anos, nota-se a necessidade do aumento e melhorias voltada às políticas públicas relacionadas à sexualidade na adolescência, na promoção e prevenção, serviço que pode ser melhorado, com ênfase, nas UBS, onde se tem acompanhamento familiar, podendo assim promover essa atenção, não apenas a gestante, mais ao companheiro e família como todo, uma vez que a equipe tem ligação direta com as famílias assistidas (Cardoso, Araújo, Cavalcante, Frota, \& Melo, 2018).

No que se refere ao perfil escolaridade, e em concordância com um estudo em Maringá - PR, sua maior incidência está presente em mulheres com Ensino Fundamental Incompleto (Ramos \& Boni, 2018). Os dados da seguinte pesquisa evidencia que o alto índice dessas gestantes apresenta grau de escolaridade de $5^{\text {a }}$ e $8^{\text {a }}$ série incompleta, totalizando 490 casos notificados, que devido ao seu baixo grau de instrução e conhecimento, as tornam mais suscetíveis à contaminação.

Sobre a idade gestacional, a presente pesquisa pode constatar que o grupo com maior índice de casos de sífilis em gestantes encontrava-se no $3^{\circ}$ trimestre da gestação, representando 41,3\% dos casos, o que consente com estudo realizado em Francisco Beltrão PR, onde $74,3 \%$ do total de mulheres com sífilis gestacional encontravam-se no $3^{\circ}$ trimestre 
(Trevisan, Bechi, Teixeira, de Araújo Marchi, \& Dalla Costa, 2018), o que reforça a importância do pré-natal, consulta humanizada voltada não apenas para a gestante, mas também para o companheiro, a realização de teste rápido e acompanhamento do tratamento para que não haja desistência ou tratamento inadequado.

$\mathrm{Na}$ variável classificação clínica, a maioria dos casos de gestantes com sífilis apresentavam-se na fase latente da doença, diferente de outro estudo (Trevisan et al., 2018), onde foi notificado maior número de casos na fase primária. De acordo com o Ministério da Saúde, a maioria das pessoas que têm sífilis desconhece estarem infectadas, e com isso há o aumento de chances de transmiti-la aos seus contatos sexuais. Realidade que ocorre devido à escassez ou ausência de sintomas, conforme o estágio da infecção (Brasil, 2019). É importante salientar que como resultados adversos da sífilis na gestação, uma grande problemática é o risco de transmissão vertical da doença e que pode ocorrer em qualquer estágio clinico da sífilis materna. Sendo assim, enfatiza-se a importância do diagnóstico precoce e tratamento imediato das gestantes, no intuito de reduzir a transmissão para o bebê e as possíveis complicações decorrentes da doença.

$\mathrm{O}$ esquema de tratamento mais utilizado pelas gestantes com sífilis nesta pesquisa foi a Penicilina em 91\% dos casos, resultado igualmente a uma pesquisa realizada em Fortaleza (Cardoso et al., 2018), e corrobora com a justificativa de que a Penicilina é a droga de primeira escolha no tratamento da sífilis, que além de ser um medicamento de baixo custo, fácil acesso e ótima eficácia, sua aplicação é realizada em unidades básicas de saúde e pode ser prescrita pela(o) enfermeira(o) conforme a lei do exercício profissional de enfermagem Lei n. ${ }^{\circ}$ 7.498/86 e o Ministério da Saúde (Brasil, 2012).

\section{Considerações Finais}

Com a realização desse estudo observou-se que é contínuo o aumento de casos de sífilis em gestantes no estado do Piauí, e que o estado necessita de apoio e planejamento para a adesão de estratégias de controle e erradicação dessa condição evitável e tratável. A sífilis caracteriza-se ainda como uma das principais IST's que necessita de intervenções precoces para a diminuição das suas consequências. Os resultados, assim como os estudos utilizados na fundamentação dessa pesquisa, refletem o cenário e a realidade atual, não só a nível estadual, mas presente em todo o país. Acredita-se que os achados dessa pesquisa irão influenciar para que haja mais vigilância e comprometimento à respeito das políticas públicas de prevenção, 
assim como na criação de novas e melhorias do assistencialismo da enfermagem acerca do tema.

\section{Referências}

Brasil (2018). Ministério da Saúde. Departamento de Doenças de Condições Crônicas e Infecções Sexualmente Transmissíveis. Protocolo Clínico e Diretrizes Terapêuticas para Prevenção da Transmissão Vertical de HIV, Sífilis e Hepatites Virais. Brasília.

Brasil (2018). Ministério da Saúde. Departamento de Doenças de Condições Crônicas e Infecções Sexualmente Transmissíveis. Boletim Epidemiológico de Sífilis. Brasília.

Brasil (2012). Ministério da Saúde. Secretaria de atenção à Saúde. Departamento de atenção básica. Atenção ao pré-natal de baixo risco. Brasília.

Brasil (2005). Ministério da Saúde. Secretaria de Vigilância em Saúde. Portaria no 33 de 14 de julho de 2005. Diário Oficial da União. Brasília.

Cardoso, ARP, Araújo, MAL, Cavalcante, MDS, Frota, MA \& Melo, SPD. (2018). Análise dos casos de sífilis gestacional e congênita nos anos de 2008 a 2010 em Fortaleza, Ceará, Brasil. Ciência \& Saúde Coletiva, 23, 563-74.

Jesus, TBDSD, Mafra, ALS, Campo, VDS, Cesarino, CB, Bertolin, DC \& Martins, MI. (2019). Sífilis em gestante e congênita: casos notificados de um município do Noroeste Paulista. Nursing (Säo Paulo), 2766-71.

Pereira, AS, Shitsuka, DM, Parreira, FJ \& Shitsuka, R. (2018). Metodologia da pesquisa científica. [e-book]. Santa Maria. Ed. UAB/NTE/UFSM. Disponível em: https://repositorio.ufsm.br/bitstream/handle/1/15824/Lic_Computacao_MetodologiaPesquisa-Cientifica.pdf?sequence $=1$.

Ramos, MG \& Boni, SM. (2018). Prevalência da sífilis gestacional e congênita na população do município de Maringá-PR. Saúde e Pesquisa, 11(3), 517-26. 
Saraceni, V, Pereira, GFM, Silveira, MF, Araujo, MAL \& Miranda, AE. (2017). Vigilância epidemiológica da transmissão vertical da sífilis: dados de seis unidades federativas no Brasil. Revista Panamericana de Salud Pública, 41, e44.

Saraceni, V \& Miranda, AE. (2012). Coverage by the Family Health Strategy and diagnosis of syphilis in pregnancy and congenital syphilis. Cadernos de saude publica, 28(3), 490-96.

Trevisan, MG, Bechi, S, Teixeira, GT, Araújo Marchi, AD \& Dalla Costa, L. (2018). Prevalência da sífilis gestacional e congênita no município de Francisco Beltrão. Espaço para Saúde, 19(2).

Vasconcelos, MIO, Oliveira, KMC, Magalhães, AHR, Guimarães, RX, Linhares, MDSC, Oliveira Queiroz, MV \& Albuquerque, IMAN. (2017). Sífilis na gestação: estratégias e desafios dos enfermeiros da atenção básica para o tratamento simultâneo do casal. Revista Brasileira em Promoção da Saúde, 29, 85-92.

\section{Porcentagem de contribuição de cada autor no manuscrito}

Francisco Samuel Andrade de Alencar Campelo - 25\%

Walber de Vasconcelos Brito Júnior - 25\%

Laurimary Caminha Veloso - 50\% 